\section{Families of compacta and some theorems on sweeping}

\section{K. B or suk (Warszawa)}

1. Families of compacta. Let $\Phi$ be a function attaching to each point $x$ of a metric space $X$ a compact subset $\Phi(x) \neq 0$ of a metric space $Y$. The function $\Phi$ is said to be upper semi-continuous (see [7], p. 32) if for every neighbourhood $V$ of $\Phi(x)$ (in $Y$ ) there exists a neighbourhood $U$ of $x$ (in $X$ ) such that.

$$
\Phi\left(x^{\prime}\right) \subset V \text { for every } x^{\prime} \in U .
$$

We shall call such a function $\Phi$ a family of compacta over $X^{1}$ ). We refer to $X$ as the basis of the family $\Phi$ and to the sets $\Phi(x)$ - as the elements of $\Phi$. The sum of all elements $\Phi(x)$ attached to the points $x$ belonging to a subset $X^{\prime}$ of $X$ we shall denote by $\Phi\left(X^{\prime}\right)$.

$$
\Phi\left(x^{\prime}\right)=\bigcup_{x \in X^{\prime}} \Phi(x) .
$$

In particular, the set $\Phi(X)$ will be called the field of the family $\Phi$. Evidently, if $X^{\prime}$ is compact then also $\Phi\left(X^{\prime}\right)$ is compact.

A family $\Phi$ will be said to be compact if its basis $X$ is compact, it will be said to be simple if its elements $\Phi(x)$ are disjoint.

Let $2^{Y}$ denote the space of all not empty subcompacta of $Y$ with metric function $\varrho$ defined by the formula of Hausdorff (see [5], p. 293):

$$
\varrho(A, B)=\max \left[\sup _{x \in A} \varrho(x, B), \sup _{y \in B} \varrho(y, A)\right] \text { for } A, B \in 2^{x} .
$$

The family $\Phi$ is a map of the basis $X$ into the space $2^{Y}$. If this map is continuous, we shall say that the family $\Phi$ is continuous ${ }^{2}$ ).

1) Compare S. Eilenberg and D. Montgomery [3], where an analogous notion appears under the name of the multi-valued continuous transformation.

2) The concept of the continuous family of compacta can be considered as a generalization of the concept of the covering in the sense of B. Eckmann [2], p. 145. Namely, if $X$ is a compact subset of the space $2 Y$, then putting $\Phi(x)=x$ for every $x \in X$ we obtain a continuous family $\Phi$ with the basis $X$, constituting a covering of the set $\Phi(X)=\bigcup \begin{aligned} & \\ & x \in X\end{aligned}(x)$. Moreover, if the elements $\Phi(x)$ are disjoint (i.e. $\Phi$ is simple), then they constitute a decomposition of the set $\Phi(X)$ in the sense of B. Eckmann [2], p. 145.
2. Examples. 1 . Let $X$ be an arbitrary space and $A$ a compactum. Setting

$$
\left.\Phi(x)=(x) \times A \subset X \times A \quad \text { for every } \quad x \in X^{3}\right),
$$

we obtain a continuous function mapping $X$ into $2^{X \times A}$. Then the field $\Phi(X)$ is identical with the set $X \times A$ and for $x \neq x^{\prime}$ the sets $\Phi(x)$ and $\Phi\left(x^{\prime}\right)$ are disjoint. It follows that the Cartesian product $X \times A$ constitutes the field of a simple and continuous family with basis $X$ and elements $\Phi(x)=(x) \times A$ homeomorphic with $A$.

2. Let $\Phi$ be a simple family over a compactum $X$. Putting

$$
\chi(p)=x \text { for every } p \in \Phi(x),
$$

we obtain a continuous mapping of the field $\Phi(X)$ of $\Phi$ onto its basis $X$. Now let us assume that every element $\Phi(x)$ of $\Phi$ is homeomorphic with a fixed compactum $A$ and that for erery $x \in X$ there exists a neighbourhood $U_{0}$ (in $X$ ) such that the Cartesian product $U_{0} \times A$ can be mapped onto the set $\Phi\left(U_{0}\right)$ by a homeomorphism $h$ satisfying the condition

$$
\chi h(x, y)=x \quad \text { for every } \quad(x, y) \in U_{0} \times A .
$$

In this case the set $\Phi(X)$ is said to be a bundle over $X$ with projection $\chi$ and the fibres $\Phi(x)$ (see [10], p. 3).

3. Let $Y$ be the space obtained from the square

$$
Q=\underset{(x, y)}{E}[|x| \leqslant 7 ;|y| \leqslant 7]
$$

by the identification of every point of the form $(0, y),|y| \leqslant 2$, with the points $(0,-y-4)$ and $(0,-y+4)$.

It is evident that set $Y$ is not homeomorphic with the square. Let $L_{x}$ denote the segment composed by all points of $Q$ with the abscisse $x$. It is evident that by this identification the segment $L_{x}$ is mapped onto a simple arc, which let us denote by $\Phi(x)$. It is clear that the function $\Phi$ defined in this manner constitutes a simple and continuous family, its basis $X$ being the interval $-7 \leqslant x \leqslant 7$ and its elements being simple ares $\Phi(x)$. Observe also that the sets $\Phi(x)$ do not constitute a bundle of fibres, because no neighbourhood of the element $\Phi(0)$ is homeomorphic with the square.

4. Retaining the notation $Q, X$ and $L_{x}$ as in example 3 , let us put $a_{0}=0$ and $a_{n}=1 / n$ for every $n=1,2, \ldots$ Let us identify in the square $Q$ every point of the form $\left(a_{n}, y\right)$ with the points $\left(a_{n},-y-4\right)$ and $\left(a_{n},-y+4\right)$ for every $n=0,1, \ldots$ It is easy to see that the space $Y$ obtained by this

3) $X \times A$ denotes the Cartesian product of $X$ and $A$. 
identification from the square $Q$ is the field of the continuous $\Phi$ with the basis $X$ and the elements $\Phi(x)$ obtained from $L_{x}$ by the same identification. Let us observe that the space $Y$ is not locally contractible ${ }^{4}$ ). To show this, let us consider the set $F_{n} \subset Q$, which is the sum of two segments: one with the ends $(1 / n, 0),(1 /(n+1), 0)$ and the other with the ends $(1 / n, 4)$ and $(1 /(n+1), 4)$. By this identification the set $F_{n}$ is transformed into a simple closed curve $C_{n} \subset Y$. It is clear that for sufficiently large $n$ the curve $C_{n}$ lies in an arbitrarily given neighbourhood of the point $y_{0} \in Y$ obtained by the identification of three points, $(0,0),(0,4)$ and $(0,-4)$. Let $U$ denote a neighbourhood of $y_{0}$ (in $Y$ ), which does not contain the point $y_{1} \in Y$ obtained by the identification of two points, $(0,2)$ and $(0,-6)$. It is easy to see that $C_{n}$ is not homotopic to zero in $U$, hence $Y$ is not locally contractible at the point $y_{0}$.

It follows from this example that local contractibility of the basis and of all elements of a simple, continuous, compact family does not imply the local contractibility of the field.

5. Consider the family $\Phi$ with the basis $0 \leqslant x \leqslant 1$ and the elements $\Phi(x)$ defined as subsets of the Euclidean 3-space $E_{3}$ by the following formulas:

$$
\begin{aligned}
& \Phi(0)=\underset{p}{E}[p=(0, t, 0) ;-1 \leqslant t \leqslant 1], \\
& \Phi(x)=\underset{p}{E}\left[p=\left(x \cos (2 \pi(1 / x+t)), \sin (2 \pi(1 / x+t)),(1-t / 4) \cdot 2^{-1 / x}\right) ; 0 \leqslant t \leqslant 1\right]
\end{aligned}
$$$$
\text { for } 0<x \leqslant 1
$$

Evidently $\Phi(0)$ is a segment, $\Phi(x)$ for $x>0$ is a simple are and $\Phi$ is a continuous, compact family. Let us show that it is simple, $i$. e. that $\Phi(x) \cap \Phi\left(x^{\prime}\right) \neq 0$ implies $x=x^{\prime}$. Since $(1-t / 4) \cdot 2^{-1 / x}>0$ for $0<x \leqslant 1$ and $0 \leqslant t \leqslant 1$, we have $\Phi(x) \cap \Phi(0)=0$ for $0<x \leqslant 1$. Suppose that $\Phi(x) \cap \Phi\left(x^{\prime}\right) \neq 0$ for some $x, x^{\prime}>0$. Then there exist two numbers $t, t^{\prime}$ such that

$$
\begin{gathered}
\quad\left(x \cos (2 \pi(1 / x+t)), \sin (2 \pi(1 / x+t)),(1-t / 4) \cdot 2^{-1 / x}\right) \\
=\left(x^{\prime} \cos \left(2 \pi\left(1 / x^{\prime}+t^{\prime}\right)\right), \sin \left(2 \pi\left(1 / x^{\prime}+t^{\prime}\right)\right), \quad\left(1-t^{\prime} / 4\right) \cdot 2^{-1} x^{\prime}\right)
\end{gathered}
$$

and that $0 \leqslant t \leqslant 1,0 \leqslant t^{\prime} \leqslant 1$. It follows that there exists an integer $k$ such that $1 / x+t=1 / x^{\prime}+t^{\prime}+k$. Since the hypotheses concerning $x, t$ and $x^{\prime}, t^{\prime}$ are symmetrical, we can assume that $t \geqslant 0$. Moreover we have $(1-t / 4) \cdot 2^{-1 / x}=\left(1-t^{\prime} / 4\right) \cdot 2^{-1 / x^{\prime}}$ and consequently

$$
2^{t} \cdot(1-t / 4)=2^{t^{\prime}+k} \cdot\left(1-t^{\prime} / 4\right) .
$$

$\left.{ }^{4}\right)$ A space $M$ is said to be locally contractible at the point $p$ if for every neigh. bourhood $U$ of $p$ there exists a neighbourhood $V$ of $p$ homotopic to zero in $U$. A space locally contractible at each of its points is said to be locally contractible.
But the function

$$
\varphi(t)=2^{t} \cdot(1-t / 4)
$$

is increasing in the interval $0 \leqslant t \leqslant 1$. Hence

$$
2^{t} \cdot(1-t / 4) \leqslant 2 \cdot(1-1 / 4)=3,2 \text { and } \quad 2^{t^{\prime}+k} \cdot\left(1-t^{\prime} / 4\right) \geqslant 2^{k} \text {. }
$$

It follows by (2) that $2^{k} \leqslant 3 / 2$, hence $k=0$ and $t=t^{\prime}$. We infer by $1, x+t=1 / x^{\prime}+t^{\prime}+k$, that $x=x^{\prime}$.

Thus the family $\Phi$ is simple. Let us observe that each of the sets $\Phi(x)$ is a simple arc and the set $\Phi(X)$ is a non-locally connected continuum. It follows that the local connectivity of the basis and of all elements of a simple, continuous and compact family does not imply the local connectivity of its field.

6 . Let $S$ denote the $(n-1)$-dimensional sphere $(n>1)$ defined in the Euclidean $n$-space $E_{n}$ by the equation

$$
x_{1}^{2}+x_{2}^{2}+\ldots+x_{n}^{2}=16 .
$$

Let us assign to every point $x \in S$ the continumm $\Phi(x)$ defined as follows:

1) If $x$ lies on the straight line joining the point $a=(0, \ldots, 0,5)$ with the centre $c=(0, \ldots, 0)$ of $S$, then $\Phi(x)$ denotes the segment $\overline{a x}$.

2) If $x$ does not lie on the straight line joining $a$ with $c$, then let us denote by $P_{x}$ the plane in $E_{n}$ spanned by the points $a, c$ and $x$. The intersection of $P_{x}$ with the $(n-1)$-dimensional hyperplane $x_{n}=0$ is a straight line containing exactly two points $p_{x}$ and $p_{x}^{\prime}$ lying at the distance 2 from $c$. Let us denote by $K_{x}$ and $K_{x}^{\prime}$ two circles in $P_{x}$ with radius 1 and with centres $p_{x}$ and $p_{x}^{\prime}$ respectirely. By $G_{x}$ and $G_{x}^{\prime}$ we denote the bounded regions determined in $P_{x}$ by $K_{x}$ and $K_{x}^{\prime}$ respectively. It is easy to see that the segment $\overline{a x}$ cuts at most one of the regions $G_{x}$ and $G_{x}^{\prime}$. It follows that either the set $\overline{a x} \cdot\left(G_{x}+G_{x}^{\prime}\right)$ is an open segment $L_{x}$ or it is empty. In the first case we denote by $C_{x}$ the eircle which lies in $P_{x}$ and for which the segment $L_{x}$ constitutes the diameter. In the second case we set $C_{x}=0$. Now we set

$$
\Phi(x)=\left(\overline{a x}+C_{x}\right)-\left(G_{x}+G_{x}^{\prime}\right) \quad \text { for every } \quad x \in S .
$$

It is easy to see that $\Phi$ is a continuous family over $S$ with elements $\Phi(x)$ being either simple arcs or 1-dimensional curves of the homotopy type $^{5}$ ) of the circle. Moreover, $x \in \Phi(x)$ for every $x \in S$. The field $\Phi(S)$ of the family $\Phi$ is a subset of $E_{n}$ such that the bounded component $G$ of the set $E_{n}-S$ is not contained in $\Phi(S)$.

5) In the sense of W. Hurewicz. See [6], p. 125. 
7. Retaining the notation used in example 6 , let us assign to every point $x \in S$ the point $f(x) \in S$ such that the common part of the straight line joining $a$ and $x$ with $S$ consists of the points $x$ and $f(x)$. It is evident that $f(x)$ is a continuous mapping of the sphere $S$ onto itself satisfying the condition $f[f(x)]=x$ for every $x \in S$. If we set

$$
\Phi_{0}(x)=[\Phi(x) \cup \Phi f(x)] \cap \bar{G} \quad \text { for every } \quad x \in S,
$$

then we obtain a continuous family $\Phi_{0}$ over $S$ with elements lying in $\bar{G}$. Moreover,

$$
x \in \Phi_{0}(x)=\Phi_{0}[f(x)] \quad \text { for every } \quad x \in S
$$

and if $x, y \in S$ and $x \neq y \neq f(x)$, then $\Phi_{0}(x) \cap \Phi_{0}(y)=0$. Let us observe that the region $G$ is not contained in the field of the family $\Phi_{0}$.

3. Families with acyclic elements. Let $X$ be a metric space. For every point $a$ of $X$ and for every $\varepsilon>0$ let us denote by $X(a, \varepsilon)$ the $\varepsilon$-neighbourhood of $a$ in $X$, i.e.

$$
X(a, \varepsilon)=\underset{x}{E}[x \in X ; \varrho(a, x)<\varepsilon] .
$$

LEMara. Let $\Phi$ be a compact family with basis $X$ and elements $\Phi(x)$ acyclic in the dimension $\left.k^{6}\right)$. There exists a positive function $\alpha_{0}(\eta)$ defined for $\eta>0$, satisfying the condition

$$
\lim _{\eta \rightarrow 0} \alpha_{0}(\eta)=0
$$

and such that for every point $a \in X$ each $k$-dimensional $\eta$-cycle lying in the eet $\Phi[X(a, \eta)]$ is $a_{0}(\eta)$-homologous to zero in the set $\Phi\left[X\left(a, a_{0}(\eta)\right)\right]$.

Proof. If such a function does not exist, then there exist a positive $\varepsilon$, a sequence of positive numbers $\left\{\eta_{i}\right\}$ converging to 0 and a sequence of points $\left\{a_{i}\right\} \subset X$ such that in the set $\Phi\left[X\left(a_{i}, \eta_{i}\right)\right]$ there exists a $k$-dimensional $\eta_{i}$-cyele $\gamma_{i}$ not $\varepsilon$-homologous to zero in $\Phi\left[X\left(a_{i}, \eta_{i}\right)\right]$. Evidently, if $\left\{n_{i}\right\}$

b) By a $k$-dimensional $\varepsilon$-simplex of $X$ we understand a set of diameter $<\varepsilon$ composed by $k+1$ different points of $X$. In the known manner one introduces the notion of an oriented $\varepsilon$-simplex of $X$, of an $\varepsilon$-chain of $X$ with arbitrarily given coefficients of an an $\varepsilon$-cycle of $X$. Two $\varepsilon$-cycles $\gamma$ and $\gamma^{\prime}$ of $X$ are said to be $\varepsilon$-homologous in $X$ (notion $\gamma_{\mathbb{e}} \gamma^{\prime}$ in $X$ ) if there exists an $\varepsilon$-chain $\psi$ of $X$, whose boundary $\partial x$ is equal to $\gamma-\gamma^{\prime}$. By a $k$-dimensional true cycle of $X$ we understand a sequence $\gamma=\left\{\gamma_{i}\right\}$, where $\gamma_{i}$ are $k$-dimensional $\varepsilon_{i}$-cykles of $X$ with $\lim _{i \rightarrow \infty} \varepsilon_{i}=0$. The $k$-dimensional true cycles of $X$ constitute a group with the group-operation defined by the formula: $\left\{\gamma_{i}\right\}+\left\{\gamma_{i}^{\prime}\right\}=\left\{\gamma_{i}+\gamma_{i}^{\prime}\right\}$. If $\gamma=\left\{\gamma_{i}\right\}$ and $\gamma^{\prime}=\left\{\gamma_{j}^{\prime}\right\}$ are two true cycles of $X$ and if there exists a sequence $\eta_{i} \rightarrow 0$ such that $\gamma_{i} \tilde{\eta}_{i} \gamma_{i}^{\prime}$ in $X$, then $\gamma$ and $\gamma^{\prime}$ are said to be homologous in $X$ (notation: $\gamma \sim \gamma^{\prime}$ in $X$ ). A compact space $X$ is said to be acyclic in the dimension $k$ if every $k$-dimensional true cycle $\gamma$ of $X$ (with arbitrary coefficients) is homologous to zero in $X$. is an increasing sequence of indices, then the sequences $\left\{a_{n_{i}}\right\}$ and $\left\{\eta_{n_{i}}\right\}$ satisfy the same conditions as $\left\{a_{i}\right\}$ and $\left\{\eta_{i}\right\}$. It follows that we can assume (since $X$ is compact) that the sequence $\left\{a_{i}\right\}$ converges to a point $a_{0} \varepsilon X$. Let us set

$$
\begin{aligned}
& A_{0}=X\left(a_{0}, \varepsilon / 2\right), \\
& A_{i}=X\left(a_{i}, \eta_{i}\right) \quad \text { for } \quad i=1,2, \ldots
\end{aligned}
$$

Since $\Phi$ is upper-semicontinuous, there exists a sequence $\left\{\beta_{i}\right\}$ of positive numbers converging to 0 and such that the set $\Phi\left(A_{i}\right)$ lies in the $\beta_{i}$-neigh bourhood of the set $\Phi\left(a_{0}\right)$, for every $i=1,2 \ldots$ It follows that $\gamma_{i}$ is $\left(\eta_{i}+2 \beta_{i}\right)$-homologous in the set $\Phi\left(A_{i}\right)+\Phi\left(a_{0}\right)$ to an $\left(\eta_{i}+2 \beta_{i}\right)$-cycle $\gamma_{i}^{\prime}$ lying in $\Phi\left(a_{0}\right)$. But $a_{i} \rightarrow a_{0}$ implies that there exists an index $i_{0}$ such that for $i>i_{0}$ it is $\varrho\left(a_{i}, a_{0}\right)<\varepsilon / 4, \eta_{i}<\varepsilon / 4$ and $\beta_{i}<\varepsilon / 4$. It follows that

$$
A_{i} \subset A_{0} \subset X\left(a_{i}, \varepsilon\right) \text { and } \eta_{i}+2 \beta_{i}<\varepsilon \text { for every } i>i_{0} .
$$

Consequently, for $i>i_{0}$, the set $\Phi\left(A_{i}\right)+\Phi\left(a_{0}\right)$ lies in the set $\Phi\left(A_{0}\right) \subset \Phi\left[X\left(a_{i}, \varepsilon\right)\right]$. Since $\eta_{i}+2 \beta_{i}<\varepsilon$, it follows that the $k$-dimensional $\left(\eta_{i}+2 \beta_{i}\right)$-cycle $\gamma_{i}^{\prime}$ is not $\varepsilon$-homologous to zero in $\Phi\left(A_{0}\right)$ and consequently it is also not $\varepsilon$-homologous to zero in the set $\Phi\left(a_{0}\right) \subset \Phi\left(A_{0}\right)$. But this is impossible, because $\eta_{i}+2 \beta_{i} \rightarrow 0$ and the set $\Phi\left(a_{0}\right)$ is acyclic in the dimension $k$.

4. Homomorphism $\vartheta$. Now we shall prove the following

LemMa. Let $\Phi$ be a family with compact basis $X$ and elements $\Phi(x)$ acyclic in the dimensions $\leqslant k$. Let $\varphi$ and $\psi$ be two continuous functions defined on $X$ and satisfying the condition

$$
\varphi(x), \psi(x) \in \Phi(x) \quad \text { for exery } x \in X .
$$

For ecery $\varepsilon>0$ there exist $a n \quad \eta>0$ and a homomorphism $\vartheta$ assigning to every r-chain * of dimension $l \leqslant k$ lying in $X$ an $(l+1)$-dimensional $\varepsilon$-chain $\vartheta(x)$ lying in $\Phi(X)$ and such that

$$
\begin{gathered}
\partial[\vartheta(x)]=\varphi(x)-\psi(x)-\vartheta(\partial x), \\
\text { if for a point } a \in X \text { the chain } x \text { lies in } X(a, \eta), \\
\text { then } \vartheta(x) \text { lies in } \Phi[X(a, \varepsilon)] .
\end{gathered}
$$

Proof. If $l=-1$ then $x$ is an element of the group of coefficients and it suffices to set $\vartheta(x)=0$. Assume now that the statement is true for chains of dimension $\leqslant k^{\prime}<k, i$. e. that there exist a positive function $\alpha_{1}(\eta)$ defined for $\eta>0$ and satisfying the condition

$$
\lim _{\eta \rightarrow 0} \alpha_{1}(\eta)=0
$$


and a homomorphism $\vartheta$ assigning to every $\eta$-chain $\varkappa$ of dimension $l \leqslant k^{\prime}$ lying in $X$ an $(l+1)$-dimensional $\alpha_{1}(\eta)$-chain $\vartheta(x)$ lying in $\Phi(X)$ and satisfying (5) and

(8) if $*$ lies in $X(a, \eta)$, then $\vartheta(\varkappa)$ lies in $\Phi\left[X\left(a, a_{1}(\eta)\right)\right]$.

Since $\varphi$ and $\psi$ are uniformly continuous on $X$, there exists a function $\beta(i)$ refined for $\eta>0$ and such that

$$
0<\eta \leqslant \beta(\eta), \quad \lim _{\eta \rightarrow 0} \beta(\eta)=0,
$$

and

if $0 \neq X^{\prime} \subset X$ and $\delta\left(X^{\prime}\right)<\eta$ then $\delta\left(\varphi\left(X^{\prime}\right)\right)<\beta(\eta)$ and $\delta\left(\varphi\left(X^{\prime}\right)\right)<\beta(\eta)$.

Now let us assign to every $l$-dimensional $\eta$-simplex $\Delta$ lying in $X$ and positively oriented ${ }^{7}$ ) one of its vertices $a_{\Delta}$. Then $\varphi(\partial \Delta)$ and $\psi(\partial \Delta)$ are $(l-1)$-dimensional $\beta(\eta)$-eycles lying in $\Phi\left[X\left(a_{\Delta}, \eta\right)\right] \subset \Phi\left[X\left(a_{\Delta}, \beta(\eta)\right)\right]$. By the lemma of section 3 there exists in $\Phi\left[X\left(a_{\Delta}, a_{0} \beta(\eta)\right)\right]$ an $l$-dimensional $\alpha_{0} \beta(\eta)$-chain $\lambda$ such that

(10)

$$
\partial \lambda=\varphi(\partial \Delta)-\psi(\partial \Delta) \text {. }
$$

By the hypothesis of induction, the $a_{1}(\eta)$-chain $\vartheta(\partial \Delta)$ lies in the set $\Phi\left[X\left(a, \alpha_{1}(\eta)\right)\right]$ and satisfies the condition

\section{Putting}

$$
\partial[\vartheta(\partial \Delta)]=\varphi(\partial \Delta)-\psi(\partial \Delta)-\vartheta(\partial \partial \Delta)=\varphi(\partial \Delta)-\psi(\partial 1) .
$$

$$
a_{2}(\eta)=\alpha_{0} \beta(\eta)+\alpha_{1}(\eta)
$$

we infer by (3) and (9) that

$$
\lim _{\eta \rightarrow 0} \alpha_{2}(\eta)=0 .
$$

Moreover, it follows by (9), (10) and (11) that $\lambda-\vartheta(\lrcorner \Delta)$ and $\lambda-\varphi(\Delta)+\varphi(\Delta)$ are $\alpha_{2}(\eta)$-cycles lying in the set $\Phi\left[X\left(a_{\Delta}, a_{2}(\eta)\right)\right]$. Applying again the lemma of section 3 we conclude that there exist in $\Phi\left[X\left(a_{4}, a_{0} a_{2}(\eta)\right)\right]$ two $(l+1)$-dimensional $a_{0} a_{2}(\eta)$-chains $\mu_{1}$ and $\mu_{2}$ such that

$$
\begin{aligned}
& \text { (13) } \\
& \text { and } \\
& a_{3}(\eta)=a_{0} a_{2}(\eta)+\eta \\
& \vartheta(\Delta)=\mu_{1}-\mu_{2} .
\end{aligned}
$$$$
\text { Now let } \quad 2 \mu_{1}=\lambda-\vartheta(\partial \Delta), \quad 2 \mu_{2}=\lambda-\varphi(\Delta)+\psi(\Delta) \text {. }
$$

7) The set of all oriented $\eta$-simplexes of $X$ ean be decomposed into two disjoint sets $P$ and $N$ such that of every pair of oppositely oriented $\eta$-simplexes $\Delta$ and $-\Delta$ one belongs to $P$ and the other to $N$. The simplexes belonging to $P$ are said to be positively oriented, and the simplexes belonging to $N$-negatively oriented.
Then $\vartheta(\Delta)$ is an $\alpha_{3}(\eta)$-chain such that

$\partial(\vartheta(\Delta))=\partial \mu_{1}-\partial \mu_{2}=\lambda-\vartheta(\partial \Delta)-\lambda+\varphi(\Delta)-\psi(\Delta)=\varphi(\Delta)-\psi(\Delta)-\vartheta(\partial \Delta)$.

Hence $\vartheta(\Delta)$ satisfies condition (5). Moreover, if $\Delta$ lies in $X(a, \eta)$, then

$$
X\left(a_{A}, \alpha_{0} \alpha_{2}(\eta)\right) \subset X\left(a, \alpha_{3}(\eta)\right)
$$

and consequently $\vartheta(\Delta)$ lies in $\Phi\left[X\left(a, a_{3}(\eta)\right)\right]$.

Finally, it follows by (3), (12) and (13) that $\lim a_{3}(\eta)=0$. Consequently there exists for every $\varepsilon>0$ an $\eta>0$ such that if $\Delta$ lies in $X(a, \eta)$ then $\vartheta(\Delta)$ lies in $\Phi[X(a, \varepsilon)]$. then

Now if $x$ is an arbitrarily given $\eta$-chain of the dimension $l=k^{\prime}+1$,

$$
\varkappa=\sum_{i=1}^{m} a_{i} \Delta_{i}
$$

where $A_{1}, \ldots, A_{m}$ are positively oriented $\eta$-simplexes of $X$. For every simplex $\Delta_{i}$ there exists in $\Phi(X)$ an $\varepsilon$-chain $\vartheta\left(\Delta_{i}\right)$ such that

Putting $\vartheta(x)=\sum_{i=1}^{m} a_{i} \vartheta\left(\Delta_{i}\right)$ we infer that

$$
\partial\left[\vartheta\left(\Delta_{i}\right)\right]=\varphi\left(\Delta_{i}\right)-\varphi\left(\Delta_{i}\right)-\vartheta\left(\partial \Delta_{i}\right) .
$$

$$
\partial[\vartheta(x)]=\varphi(x)-\varphi(x)-\vartheta(\partial x) .
$$

Moreover, if for a point $a \in X$ the chain $*$ lies in $X(a, \eta)$, then $\vartheta\left(\Delta_{i}\right)$ lies in $\Phi[X(a, \varepsilon)]$ for $i=1,2, \ldots, m$ and consequently $\vartheta(\%)$ also lies in $\Phi[X(a, \varepsilon)]$. This completes the proof of the lemma.

\section{Homologies in families with acyclic elements. Now we} can establish the following

THEOREM. Let $\Phi$ be a family with compact basis $X$ and elements $\Phi(x)$ acyclic in the dimensions $\leqslant k$. Let $\varphi$ and $\psi$ be two continuous functions defined on $X$ and satisfying the condition

$$
\varphi(x), \psi(x) \epsilon \Phi(x) \quad \text { for every } x \epsilon X .
$$

For. every $k$-dimensional true cycle $r=\left\{\gamma_{i}\right\}$ lying on $X$ the true cycles $\varphi(\gamma)=\left\{\varphi\left(\gamma_{i}\right)\right\}$ and $\psi(\gamma)=\left\{\psi\left(\gamma_{i}\right)\right\}$ are homologous in $\Phi(X)$.

Proof. By the lemma of section 4 there exists for every $\varepsilon>0$ an $\eta>0$ and a homomorphism $\vartheta$ assigning to every $\eta$-chain of dimension $\leqslant k$ lying in $X$ an $\varepsilon$-chain $\vartheta(x)$ lying in $\Phi(X)$ and satisfying (5). But $\gamma=\left\{\gamma_{i}\right\}$ is a true cycle of $X$. Hence there exists an index $n_{\eta}$ such that

$$
\gamma_{i} \text { is an } \eta \text {-cykle for every } i>n_{\eta} \text {. }
$$

It follows that $\hat{\partial}\left[\vartheta\left(\gamma_{i}\right)\right]=\varphi\left(\gamma_{i}\right)-\psi\left(\gamma_{i}\right)$ for every $i>n_{\eta}$. Hence $\varphi\left(\gamma_{i}\right) \sim \tilde{e} \psi\left(\gamma_{i}\right)$ in $\Phi(X)$ for every $i>n_{\eta}$. This shows that $\varphi(\gamma) \sim \psi(\gamma)$ in $\Phi(X)$. 
COROLLARY 1. Let $\Phi$ be a family with compact basis $X$ and elements acyclic in the dimensions $\leqslant k$. Suppose that there exists a point $a_{0}$ common to all elements $\Phi(x)$. Then for every continuous function $\varphi$ defined on $X$ and satisfying the condition

$$
\varphi(x) \in \Phi(x) \quad \text { for every } x \in X
$$

and for every true cycle $\gamma$ of $X$ of dimension $\leqslant k$ the true cycle $\varphi(\gamma)$ is homologous to zero in $\Phi(X)$.

Proof. It suffices to apply the last theorem, putting $\psi(x)=a_{0}$ for every $x \in X$.

COROLLARY 2. Let $X$ be a compact subset of the Euclidean n-space $E_{n}$ and $a_{0}$ a fixed point of $E_{n}$. Let $\Phi$ be a family with basis $X$ and acyclic elements ${ }^{8}$ ) satisfying the condition

$$
a_{0}, x \in \Phi(x) \subset E_{n} \quad \text { for every } x \in X
$$

Then every bounded component of $E_{n}-X$ is contained in $\Phi(X)$.

Proof. Let $p$ be a point lying in a bounded component $G$ of $E_{n}-X$ and $q$ a point lying in the unbounded component $G^{\prime}$ of $E_{n}-X$. Then there exists (see [9], p. 912) in $X$ an $(n-1$ )-dimensional true cycle $\gamma$ modulo 2 not homologous to zero in the set $E_{n}-(p)-(q)$. By corollary 1 (where we put $\varphi(x)=x$ for every $x \in X$ ) the true cycle $\gamma$ is homologous to zero in $\Phi(X) \subset E_{n}-(q)$. It follows that $p \in \Phi(X)$.

Remark 1 . If $n=2$ and if the family $\Phi$ is continuous, the statement of corollary 2 is equivalent to a theorem on sweeping, due to S. Goląb [4]. A particular case of corollary 2 (for arbitrary $n$ ) is also contained in my paper [1].

Remark 2. Example 6 of section 2 shows that in corollary 2 the hypothesis of the acyclicity of elements in all dimensions cannot be omitted.

6. Families of $(n-1)$-dimensional spheres in $E_{n}$. We shall establish a theorem constituting a generalization of a theorem on sweeping due to $F$. Leja. First we shall prove the following

Lemana. Let $\Phi$ be a compact family with basis $X$ and with elements $\Phi(x)$ lying in the Euclidean $n$-space $E_{n}(n>1)$. Suppose that all elements $\Phi(x)$ are closed $(n-1)$-dimensional manifolds (in the classical sense) and that there exist a point $a_{0} \in E_{n}$ lying for every $x \in X$ in the bounded component $G_{x}$ of $E_{n}-\Phi(x)$. Then the family $\Phi$ is continuous and so is also the family $T$ defined by the formula

$$
\Gamma(x)=\Phi(x)+G_{x} \quad \text { for every } \quad x \in X .
$$

') A compactum $F$ is said to be acyclic if it is acyclic in all dimensions. See footnote $\left.{ }^{6}\right)$.

Proof. Let $x_{0} \in X$ and $\varepsilon>0$. Since $\Phi\left(x_{0}\right)$, as a manifold, is an ANR- set ${ }^{9}$ ), there exist two neighbourhoods $U$ and $V$ of $\Phi\left(x_{0}\right)$ in $E_{n}$ and a retraction $r$ of $U$ to $\Phi\left(x_{0}\right)$ such that

$$
\begin{aligned}
& a_{0} \in E_{n}-U \subset E_{n}-\nabla, \\
& \varrho(x, r(x))<\varepsilon \text { for every } x \in V, \\
& \text { the segment } \overline{x r(x)} \text { lies in } U \text { for every } x \in V .
\end{aligned}
$$

Since $\Phi$ is upper semi-continuous there exists a positive $\eta$ such that for every $x^{\prime} \in X\left(x_{0}, \eta\right)$ we have $\Phi\left(x^{\prime}\right) \subset V$.

Let $\gamma^{\prime}$ be an $(n-1)$-dimensional true cycle modulo 2 in $\Phi\left(x^{\prime}\right)$ not homologous to zero in $\Phi\left(x^{\prime}\right)$. The retraction $r$ maps $\gamma^{\prime}$ onto a true cycle $r\left(\gamma^{\prime}\right)$ in $\Phi\left(x_{0}\right)$. Since $\overline{x r(x)} \subset U$ for every $x \in \nabla$, we have $r\left(\gamma^{\prime}\right) \sim \gamma^{\prime}$ in $U$. It follows that $r\left(\gamma^{\prime}\right) \neq 0$ in $E_{n}-\left(a_{0}\right)$ and consequently $r\left(\gamma^{\prime}\right) \neq 0$ in $\Phi\left(x_{0}\right)$. Consequently $r[\Phi(x)]=\Phi\left(x_{0}\right)$. But the distance between $\Phi\left(x^{\prime}\right)$ and $r\left[\Phi\left(x^{\prime}\right)\right]$ is $<\varepsilon$ and consequently also the distance between $\Phi\left(x_{0}\right)$ and $\Phi\left(x^{\prime}\right)$ is $<\varepsilon$. Hence the family $\Phi$ is continuous.

Moreover, if a point $p \in E_{n}$ lies at a distance $>\varepsilon$ from $\Gamma\left(x_{0}\right)$, then

$$
r^{\prime} \sim r\left(r^{\prime}\right) \sim 0 \text { in } E_{n}-(p) .
$$

Since $\gamma^{\prime}+0$ in $\Phi\left(x^{\prime}\right)$, it follows that $p \dot{\epsilon} \Gamma\left(x^{\prime}\right)$. Similarly, if a point $p^{\prime} \in E_{n}$ lies at a distance $>\varepsilon$ from $\Gamma\left(x^{\prime}\right)$, then

$$
r\left(r^{\prime}\right) \sim \gamma^{\prime} \sim 0 \text { in } E_{n}-(p) .
$$

Since $r\left(\gamma^{\prime}\right)+0$ in $\Phi\left(x_{0}\right)$, it follows that $p^{\prime} \xi \Gamma\left(x_{0}\right)$. Thus it is shown that the distance of Hausdorff between $\Gamma\left(x_{0}\right)$ and $\Gamma\left(x^{\prime}\right)$ is $<\varepsilon$ and consequently the family $\Gamma$ is continuous.

7. Generalization of Leja's theorem on sweeping. Now we shall prove the following

THEOREM. Let $X$ be a compact subset of the Euclidean n-space $E_{n}$ (11 $>1$ ) and $a_{0}$ a point belonging to the unbounded component of $E_{n}-X$ Let $\Phi$ be a family over $X$ with elements $\Phi(x)$ satisfying, for every $x \in X$, the following conditions:

$1^{\circ} \Phi(x)$ is homeomorphic to the $(n-1)$-dimensional sphere,

$2^{\circ} x \in \Phi(x) \subset E_{n}$,

$3^{\circ} a_{0}$ belongs to the bounded component of $E_{n}-\Phi(x)$. $\Phi(X)$.

Then each bounded component of the set $E_{n}-X$ is a subset of the field

9) By an ANR-set one understands a metric compact space $M$ such that if $N$ is a metric space and $M I^{\prime}$ a subset of $N$ homeomorphic with $M$, then there exist a neighbourhood $U$ of $M^{\prime}$ in $N$ and a continuous function $r$ mapping $U$ onto $M^{\prime}$ in such a manner that for everT $x \in M^{\prime}$ it is $r(x)=x$. The function $r$ is said to be a retraction of $U$ to $M^{\prime}$ 
Proof. If we adjoin to $E_{n}$ a point in the infinity $a_{\infty}$, we obtain a space $S_{n}^{\prime}$ homeomorphic with the $n$-dimensional sphere $S_{n}$. Let $\Gamma(x)$ denote the sum of $\Phi(x)$ and the bounded component $G_{x}$ of the set $E_{n}-\Phi(x)$ and let $\Gamma^{\prime}(x)=S_{n}^{\prime}-G_{x}$. By the lemma of section 6 we infer that $\Gamma$ and $\Gamma^{\prime}$ are compact and continuous families with acyclic elements such that

$$
a_{0} \in \Gamma(x) \quad \text { and } \quad a_{\infty} \in \Gamma^{\prime}(x) \text { for every } x \in X \text {. }
$$

Let us suppose that there exists a point $p$ lying in a bounded component of $E_{n}-X$ and not belonging to $\Phi(X)$. Then there exists a component $X_{0}$ of $X$ such that $p$ lies in a bounded component of $E_{n}-X_{0}$. Let us show that

$$
p \in \Gamma(x) \text { for every } x \in X_{0} .
$$

By corollary 2 of section 5 there exists a point $x_{0} \in X_{0}$ such that $p \in \Gamma\left(x_{0}\right)$. Let us decompose $X_{0}$ into two disjoint sets $X_{0}^{\prime}$ and $X_{0}^{\prime \prime}$, the first composed by all $x \in X_{\mathbf{0}}$ such that $p \in \Gamma(x)$. Since $\Gamma$ is continuous and $p \in E_{n}-\Phi(x)$, the set $X_{0}^{\prime \prime}$ is also compact. Hence the continuum $X_{0}$ is decomposed into two compact, disjoint sets $X_{0}^{\prime}$ and $X_{0}^{\prime \prime}$. Since $X_{0}^{\prime} \neq 0$ it follows that $X_{0}^{\prime \prime}=0, i$.e relation (15) is proved.

Now consider the family $\Gamma^{\prime}$, whose elements lie in the space $E_{n}^{\prime}=S_{n}^{\prime}-\left(a_{0}\right)$. Applying corollary 2 of section 5, we conclude that there exists a point $x_{1} \in X_{0}$ such that $p \in \Gamma^{\prime}\left(x_{1}\right)$. By (15) it follows that $p \in \Gamma\left(x_{1}\right) \cap \Gamma^{\prime}\left(x_{1}\right)=\Phi\left(x_{1}\right)$, which is contrary to the assumption that $p \epsilon \Phi(X)$. Thus the theorem is proved.

In the case where $n=2$ and $X$ is a simple closed curve, and under the hypothesis that the family $\Phi$ is continuous (by the lemma of section 6 this last hypothesis is superfluous) the statement of the theorem is equivalent to the theorem of F. Leja on sweeping (see [8], p. 422).

8. Involutions. Let $X$ be a metric space. A continuous mapping $f$ of $X$ into itself satisfying the condition

$$
f f(x)=x \quad \text { for every } \quad x \in X
$$

is called an involution on $X$. Evidently every involution on $X$ is a homeomorphism mapping of $X$ onto itself.

A metric $\varrho$ in $X$ will be said to be consistent with an involution $f$ m $X$ if

$$
\varrho(x, y)=\varrho(f(x), f(y)) \quad \text { for every } \quad x, y \in X .
$$

If $f$ is an arbitrarily given involution on $X$, then setting

$$
\bar{\varrho}(x, y)=\max [\varrho(x, y), \varrho(f(x), f(y))] \text { for every } x, y \in X
$$

we obtain a metric $\bar{\varrho}$ consistent with $f$ and topologically equivalent to $\varrho$. It follows that we can always assume that the metric $\varrho$ in $X$ is consistent with an arbitrarily given involution.

Examples. 8. Let $S$ be the $(n-1)$-dimensional sphere defined in $E_{n}$ by the equation

$$
x_{1}^{2}+x_{2}^{2}+\ldots+x_{n}^{2}=1 \text {. }
$$

For every integer $l$ such that $0 \leqslant l \leqslant n$ let us set

$$
\begin{gathered}
f_{l}\left(x_{1}, \ldots, x_{n}\right)=\left(y_{1}, \ldots, y_{n}\right) \text { where } y_{i}=-x_{i} \text { for } 1 \leqslant i \leqslant l \\
\text { and } y_{i}=x_{i} \text { for } i>l .
\end{gathered}
$$

It is clear that $f_{l}$ is an involution on $S$ and the Euclidean metric in $S$ is consistent with $f_{l}$. The involution $f_{l}$ is the symmetry relative to the hyperplane $H_{l}$ defined by the equations

$$
x_{i}=0 \text { for } i=1,2, \ldots, l \text {. }
$$

In particular, $f_{0}$ is the identity and $f_{n}$ the so called antipodal mapping. Evidently the degree of $f_{l}$ is equal to $(-1)^{l}$.

9. Let $X$ be the Cartesian product of the spaces $X_{1}, X_{2}, \ldots, X_{m}$ and let $f_{i}$ be an involution on $X_{i}$. Setting

(16) $f\left(x_{1}, \ldots, x_{m}\right)=\left(f_{1}\left(x_{1}\right), \ldots, f_{m}\left(x_{m}\right)\right)$ for every $\left(x_{1}, \ldots, x_{m}\right) \in X$,

we obtain an involution on $X$. In particular, if $X_{i}$ are spheres then the formula (16) gives an involution of a generalized torus.

As a simple application of the theorem of section 5 we obtain the following

THEOREM. Let $X$ be a compact space, $f$ an involution on $X$ and $\Phi$ a family over $X$ with elements $\Phi(x)$ acyclic in the dimensions $\leqslant k$ and satisfying the condition.

$$
x, f(x) \in \Phi(x) \quad \text { for every } \quad x \in X .
$$

Then every true cycle $r$ in $X$ of the dimension $\leqslant k$ is homologous in $\Phi(X)$ with $f(r)$.

Proof. It suffices to apply the theorem of section s setting $\varphi(x)=x$ and $\psi(x)=f(x)$ for every $x \in X$.

CoROLLARY. Let $f$ be an involution on a compact subset $X$ of the Euclidean $n$-space $E_{n}$ and $r$ an $(n-1)$-dimensional true cycle in $X$ such that

$$
r \sim f(r) \text { in } X
$$

Let $\Phi$ be a family over $X$ with acyclic elements satisfying the condition

$$
x, f(x) \in \Phi(x) \subset E_{n} \quad \text { for every } x \in X .
$$

Then the field $\Phi(X)$ contains at least one bounded component of $E_{n}-X$. 
Proof. Since $\tau+f(r)$ in $X$, we infer (see [9], p. 912) that there exists a bounded component $G$ of $E_{n}-X$ such that for every point $p \in G$ the true cycle $\gamma-f(\gamma)$ is not homologous to zero in $E_{n}-(p)$. But the last theorem implies that $\gamma-f(\gamma) \sim 0$ in $\Phi(X) \subset E_{n}$. Hence $p \in \Phi(X)$ for every $p \in G$, i. e. $G \subset \Phi(X)$.

In particular, we infer that if $\Phi$ is a family over the $(n-1)$-dimensional sphere $S \subset E_{n}$ with acyclic elements satisfying the condition

$$
x, f_{l}(x) \in \Phi(x) \subset E_{n} \quad \text { for every } x \in S
$$

(we use here the notation of example 8), then for odd $l$ the bounded component $G$ of $E_{n}-S$ is contained in $\Phi(X)$. In fact, if $\gamma$ is the true cycle constituting the basis for $(n-1)$-dimensional homology in $S$, then $f_{l}(\gamma)$ is not homologous with $\gamma$ in $X$. By the last corollary we infer that $G \subset \Phi(X)$.

9. Families consistent with an involution. We shall say that a family $\Phi$ over $X$ is consistent with an involution $f$ on $X$ if

$$
\Phi(x)=\Phi[f(x)] \quad \text { for every } \quad x \in X .
$$

As we have already remarked, we can assume without loss of generality that the metric $\varrho$ in $X$ is consistent with the given involution $f$. Under this assumption let us prove the following

LEMMA. Let $f$ be an involution on a compact space $X$ and $\Phi$ a family over $X$ with elements acyclic in the dimensions $\leqslant k$ consistent with $f$. Let $\varphi$ be a continuous mapping defined on $X$ and satisfying the condition.

$$
\varphi(x) \epsilon \Phi(x) \text { for every } x \in X .
$$

Then for every $\varepsilon>0$ there exist an $\eta>0$ and a homomorphism $\vartheta_{0}$ assigning to every $\eta$-chain $x$ of dimension $l \leqslant k$ lying in $X$ an $(l+1)$-dimensional $\varepsilon$-chain $\vartheta_{0}(x)$ lying in $\Phi(X)$ and such that

$$
\begin{gathered}
\partial\left[\vartheta_{0}(x)\right]=\varphi(x)-\varphi f(x)-\vartheta_{0}(\partial x), \\
\vartheta_{0}(x)=-\vartheta_{0}[f(x)],
\end{gathered}
$$

if for a point $a \in X$ the chain $*$ lies in $X(a, \eta)$,

then $\vartheta_{0}(x)$ lies in $\Phi[X(a, \varepsilon)]$.

Proof. If $l=-1$ then we set $\vartheta_{0}(x)=0$. Assume now that the statement is true for chains of dimension $\leqslant k^{\prime}<k$, i. e. that there exists a pasitive function $a_{4}(\eta)$ defined for $\eta>0$ and satisfying the condition

$$
\lim _{\eta \rightarrow 0} \alpha_{4}(\eta)=0
$$

and that there exists a homomorphism $\vartheta_{0}$ assigning to every $\eta$-chain $*$ of the dimension $l \leqslant k^{\prime}$ lying in $X$ an $(l+1)$-dimensional $\alpha_{4}(\eta)$-chain $\vartheta_{0}(x)$ lying in $\Phi(X)$ and satisfying conditions (17), (18) and (19), with $\varepsilon=\alpha_{4}(\eta)$. Consider now, for every $\eta$-simplex $\Delta$ of $X$, the set $\{\Delta\}$ of four $\eta$-simplexes $\Delta,-\Delta, f(\Delta), f(-\Delta)$. Evidently two sets $\{\Delta\}$ and $\left\{\Delta^{\prime}\right\}$ are either identical or disjoint. By the axiom of choice there exists a class $\Sigma$ containing exactly one simplex of erery set $\{\Delta\}$.

Let $l=k^{\prime}+1$, let $\Delta$ be an $l$-dimensional simplex belonging to $\Sigma$ and let $a_{\Delta}$ be one of its vertices. Let $\alpha_{0}(\eta)$ be a positive function satisfying the statement of the lemma of section 3 . Then there exists in the set $\Phi\left[X\left(a_{\Delta}, a_{0}(\eta)\right)\right]$ an $l$-dimensional $\alpha_{0}(\eta)$-chain $\lambda$ such that

$$
\partial \lambda=\varphi(\partial \Delta)-\varphi f(\partial \Delta) \text {. }
$$

Moreover, we can assume that in the case of $\Delta=f(\Delta)$ we have $\lambda=0$.

By the inductive hypothesis the homomorphism $\vartheta_{0}$ is defined already on $\partial \Delta$ and the $a_{4}(\eta)$-chain $\vartheta_{0}(\partial \Delta)$ lies in the set $\Phi\left[X\left(a_{\Delta}, a_{4}(\eta)\right)\right]$ and satisfies the following condition:

$$
\partial\left[\vartheta_{0}(\partial \Delta)\right]=\varphi(\partial \Delta)-\varphi f(\partial \Delta)-\vartheta_{0}(\partial \partial \Delta)=\varphi(\partial \Delta)-\varphi f(\partial \Delta) .
$$

Let us set

Then the chains

$$
\alpha_{5}(\eta)=\alpha_{0}(\eta)+\alpha_{4}(\eta)
$$

$$
\lambda-\vartheta_{0}(\partial \Delta) \quad \text { and } \quad \lambda-\varphi(\Delta)+\varphi f(\Delta)
$$

are $l$-dimensional $\alpha_{5}(\eta)$-cycles lying in $\Phi\left[X\left(a_{\Delta}, \alpha_{5}(\eta)\right)\right]$. Applying the lemma of section 3 we infer that there exist in the set $\Phi\left[X\left(a_{4}, a_{0} a_{5}(\eta)\right)\right]$ two $(l+1)$-dimensional $\alpha_{0} \alpha_{5}(\eta)$-chains $\mu_{1}$ and $\mu_{2}$ such that

$$
\partial \mu_{1}=\lambda-\vartheta_{0}(\partial \Delta), \quad \partial \mu_{2}=\lambda-\varphi(\Delta)+\varphi f(\Delta) .
$$

Moreover, we can assume that in the case of $\lambda-\vartheta_{0}(\partial \Delta)=\lambda-\varphi(\Delta)+\varphi f(\Delta)=0$ we have $\mu_{1}=\mu_{2}=0$. Setting

$$
\alpha_{6}(\eta)=\alpha_{0} \alpha_{5}(\eta)+\eta
$$

$$
\vartheta_{0}(\Delta)=\mu_{1}-\mu_{2}
$$

we obtain an $\alpha_{6}(\eta)$-chain $\vartheta_{0}(\Delta)$ such that

$$
\partial\left[\vartheta_{0}(\Delta)\right]=\hat{\delta} \mu_{1}-\partial \mu_{2}=\varphi(\Delta)-\varphi f(\Delta)-\vartheta_{0}(\partial \Delta) .
$$

Moreover, if $\Delta$ lies in $X(a, \eta)$, then

$$
\bar{X}\left(a_{\Delta}, \alpha_{0} \alpha_{5}(\eta)\right) \subset X\left(a, \alpha_{6}(\eta)\right)
$$

and consequently $\vartheta_{0}(\Delta)$ lies in $\Phi\left[X\left(a, \alpha_{6}(\eta)\right)\right]$. 
Thus the operation $\vartheta_{0}$ is defined for all $l$-dimensional $\eta$-simplexes $\Delta$ belonging to $\Sigma$. Setting

$$
\vartheta_{0}(-\Delta)=-\vartheta_{0}(\Delta)
$$

we extend the operation $\vartheta_{0}$ to the simplexes $-\Delta$ such that $\Delta \epsilon \Sigma$. Eridently conditions (17) and (18) are satisfied. Finally, we put

$$
\vartheta_{0}[f(\Delta)]=-\vartheta_{0}(\Delta)
$$

if $\Delta$ or $-\Delta$ belongs to $\Sigma$. If $\Delta=f(\Delta)$, then $\lambda=0$ and

$$
\lambda-\vartheta_{0}(\partial \Delta)=-\vartheta_{0}[\partial f(\Delta)]=-\vartheta_{0}[f(\partial \Delta)]=\vartheta_{0}(\partial \Delta) .
$$

Hence the chain $\vartheta_{0}(\partial \Delta)$ (with integral coefficients) is equal to its opposite $-\vartheta_{0}(\partial \Delta)$. It follows that $\partial \mu_{1}=\lambda-\vartheta_{0}(\partial \Delta)=0$ and also that $\partial \mu_{2}=\lambda-\varphi(\Delta)-$ $-\varphi[f(\Delta)]=0$. By the hypothesis made by the construction of the chains $\mu_{1}$ and $\mu_{2}$ we have in this case $\mu_{1}=\mu_{2}=0$ and consequently also $\vartheta_{0}(\Delta)=$ ? It follows by (26) and (27) that in this case $\vartheta_{0}[f(\Delta)]=-\vartheta_{0}(\Delta)=\vartheta_{0}(\Delta)=0$.

If, however, $f(\Delta)=-\Delta$, then the value of $\vartheta_{0}$ on $f(\Delta)$ given by formula (27) is consistent with the value given by formula (26). Hence formula (27) does not lead to a contradiction.

Let us show that the operation $\vartheta_{0}$ defined in this manner for all $\eta$-simplexes satisfies conditions (17), (18) and (19). If $\Delta \epsilon \Sigma$, relation (17) is a consequence of (25) and relation (18) is a consequence of (27). Relation (19) follows by (26) and the definition of $\mu_{1}$ and $\mu_{2}$. It follows also by (24) that relations (17), (18) and (19) are satisfied if $-\Delta \epsilon \Sigma$. Finally if $(17),(18)$ and (19) are satisfied for a simplex $\Delta$, then we infer by (27) that

$$
\begin{gathered}
\partial\left[\vartheta_{0}(f(\Delta))\right]=-\partial\left[\vartheta_{0}(\Delta)\right]=-\varphi(\Delta)+\varphi[f(\Delta)]+\vartheta_{0}(\partial \Delta) \\
=-\varphi[f f(\Delta)]+\varphi[f(\Delta)]-\vartheta_{0}[f(\partial \Delta)]=\varphi[f(\Delta)]-\varphi[f f(\Delta)]-\vartheta_{0}[\partial f(\Delta)],
\end{gathered}
$$

i. e. the chain $f(\Delta)$ satisfies (17). Condition (18) follows immediately by (27). Finally, if for a point $a \in X$ the simplex $f(\Delta)$ lies in $X(a, \eta)$, then $\Delta$ lies in $X[f(a), \eta]=f[X(a, \eta)]$ (since the metric $\varrho$ is consistent with $f$ ). Then $\vartheta_{0}(\Delta)=-\vartheta_{0}[f(\Delta)]$ lies in $\Phi\left[X\left(f(a), a_{6}(\eta)\right)\right]=\Phi\left[f\left(X\left(a, a_{6}(\eta)\right)\right)\right]$ and consequently condition (19), for $\varepsilon=\alpha_{6}(\eta)$, is satisfied.

Now if $\varkappa$ is an arbitrary $\eta$-chain in $X$ of the dimension $l=k^{\prime}+1$, then $x=\sum_{i=1}^{m} a_{i} \Delta_{i}$ where $\Delta_{1}, \Delta_{2}, \ldots, \Delta_{m}$ are $\eta$-simplexes in $X$. For every $\Delta_{i}$ the $\alpha_{6}(\eta)$-chain $\vartheta_{0}\left(\Delta_{i}\right)$ (with integral coefficients) lying in $\Phi(X)$ and such

$$
\partial\left[\vartheta_{0}\left(\Delta_{i}\right)\right]=\varphi\left(\Delta_{i}\right)-\varphi f\left(\Delta_{i}\right)-\vartheta_{0}\left(\partial \Delta_{i}\right), \quad \vartheta_{0}\left(\Delta_{i}\right)=-\vartheta_{0}\left[f\left(\Delta_{i}\right)\right]
$$

is already defined.
Putting

we infer that

$$
\vartheta_{0}(\varkappa)=\sum_{i=1}^{m} a_{i} \vartheta_{0}\left(\Delta_{i}\right)
$$

$$
\partial\left[\vartheta_{0}(x)\right]=\varphi(x)-\varphi f(x)-\vartheta_{0}(\partial x) \quad \text { and } \quad \vartheta_{0}(x)=-\vartheta_{0}[f(x)] .
$$

Moreover, if for a point $a \epsilon X$ the chain $x$ lies in $X(a, \eta)$, then $\vartheta_{0}\left(\Delta_{i}\right)$ lies in $\Phi\left[X\left(a, a_{6}(\eta)\right)\right]$ for $i=1,2, \ldots, m$ and consequently $\vartheta_{0}(x)$ lies in $\Phi\left[X\left(a, a_{6}(\eta)\right)\right]$. To obtain the statement of the lemma it suffices to observe that $\lim _{\eta \rightarrow 0} \alpha_{6}(\eta)=0$.

$\lim _{\eta \rightarrow 0}$

10. Triangulations consistent with an involution. Consider now the case where $X$ is a polytope (in general, curvilinear). If $T$ is a triangulation of $X$, then we shall say that an $\varepsilon$-simplex $\Delta=\left(a_{0}, a_{1}, \ldots, a_{k}\right)$ belongs to $T$ if there exists in $T$ a geometrical simplex with vertices $a_{0}, a_{1}, \ldots, a_{k}$. A triangulation $T$ of $X$ will be said to be consistent with an involution $f$ on $X$ if for every $\varepsilon$-simplex $\Delta=\left(a_{0}, a_{1}, \ldots, a_{k}\right)$ belonging to $T$ the simplex $f(\Delta)=\left(f\left(a_{0}\right), f\left(a_{1}\right), \ldots, f\left(a_{k}\right)\right)$ also belongs to $T$ and moreover the equality $\Delta= \pm f(\Delta)$ implies $f\left(a_{i}\right)=a_{i}$ for every $i=0,1, \ldots, k$.

Example 10. Let $X$ be the Euclidean $(n-1)$-dimensional unit sphere $S$ in $E_{n}$ with centre $c_{0}=(0, \ldots, 0)$ and let $f_{l}$ be (as in example 8 ) the symmetry relative to the hyperplane $H_{l}$ defined by the equations $x_{i}=0, i=1,2, \ldots, l$. The hyperplane $H^{\prime}$ defined by the equation $x_{n}=0$ intersects $S$ along the $(n-2)$-dimensional unit sphere $S^{\prime}$ (called equator of $S)$. Putting

$$
f_{l}^{\prime}(x)=f_{l}(x) \quad \text { for every } \quad x \in S^{\prime},
$$

we obtain an involution on $S^{\prime}$ constituting also the symmetry relative to $H_{l}$. Let us assume that there exists a triangulation $T^{\prime}$ of $S^{\prime}$ (with spherical simplexes on $S^{\prime}$ ) consistent with $f_{l}^{\prime}$; for $n=1$ the existence of a such triangulation of $S^{\prime}$ is evident. Let us denote by $p$ and $p^{\prime}$ two points of $S$ (poles of $S$ ) of the form

$$
p=(0, \ldots, 0,1), \quad p^{\prime}=(0, \ldots, 0,-1) .
$$

Putting for every simplex $\Delta^{\prime}=\left(a_{0}, \ldots, a_{k}\right)$ of $T^{\prime}$

$$
p \Delta^{\prime}=\left(p, a_{0}, \ldots, a_{k}\right), \quad p^{\prime} \Delta^{\prime}=\left(p^{\prime}, a_{0}, \ldots, a_{k}\right)
$$

we easily see that the simplexes $p \Delta^{\prime}, p^{\prime} \Delta^{\prime}$ and their faces constitute a triangulation $T$ of $S$ consistent with $f_{l}$. It is easy to see that by the iterated process of barycentric subdivision we can obtain from $T$ a triangulation of $S$ consistent with $f_{l}$ and having all simplexes with arbitrarily small diameter.

Fundamenta Mathematicae. T. XLIr. 
THEOREM. Let $f$ be an involution (different from the identity) on an (n-1)-dimensional (curvilinear) pseudo-manifold $X$ lying in the Euctidean $n$-space $E_{n}$ and let $\left\{T_{y}\right\}$ be a sequence of triangulations of $X$ consistent with $f$ and such that the diameters of simplexes of $T_{v}$ converge with $1 / \nu$ to zero. Moreover, let $\Phi$ be a family over $X$ consistent with the involution $f$, with elements $\Phi(x)$ acyclic and satisfying the condition.

$$
x \in \Phi(x) \subset E_{n} \quad \text { for every } x \in X \text {. }
$$

Then the bounded component of $E_{n}-X$ is contained in the field $\Phi(X)$.

Proof. The $(n-1)$-dimensional pseudo-manifold $X$, as lying in $E_{n}$, is orientable. Let us choose an orientation of $X$ and let $\Delta_{v 1}, \Delta_{v 2}, \ldots, \Delta_{v_{m_{r}}}$ be all $(n-1)$-dimensional simplexes of $T_{v}$ oriented positively. Then the chains

$$
\gamma_{v}=\Delta_{v 1}+\Delta_{v 2}+\ldots+\Delta_{v m_{v}}
$$

constitute a true cycle $\gamma=\left\{\gamma_{\nu}\right\}$ not homologous to zero on $X$. If the involution $f$ changes the orientation of $X$, then $\gamma+f(\gamma)$ in $X$ and we infer by the corollary of section 8 that $\Phi(X)$ contains the bounded component of $E_{n}-X$.

Suppose now that the involution $f$ does not change the orientation of $X$. Since $T_{v}$ is consistent with $f$, we conclude that $f\left(\gamma_{v}\right)=\gamma_{y}$, and consequently

$$
f\left(\Delta_{v i}\right)=\Delta_{v j(i)} \quad \text { for } \quad i=1,2, \ldots, m_{\nu},
$$

where $j(i)$ depends on $i$. Let us denote by $\lambda_{v}$ the sum of all $\Delta_{v i}$ such that $j(i)=i$. Then $\gamma_{\nu}-\lambda_{\nu}$ can be decomposed into the sum of two chains $*$ and $f(x)$ with different simplexes. Hence

and

$$
\gamma_{p}=x_{p}+f\left(x_{v}\right)+\lambda_{r}
$$

$$
\partial x_{v}+\partial f\left(x_{v}\right)+\partial \lambda_{v}=0 .
$$

Moreover, let us observe that the hypothesis that $f$ is not the identity implies that for almost all $v$ we have $\varkappa_{p} \neq 0$ and also

$$
x_{v}-f\left(\varkappa_{p}\right) \neq 0 \bmod 2 \text {. }
$$

Let us observe that $\partial \lambda_{p}=0$. Otherwise there would exist an $(n-2)$-dimensional simplex $\Delta$ appearing with the coefficient 1 on the boundary of a simplex $\Delta_{v i}$ belonging to $\lambda_{v}$ and with the coefficient -1 on the boundary of a simplex $\Delta_{v i^{\prime}}$ belonging to $x_{y}$ or to $f\left(\varkappa_{v}\right)$. Hence $\Delta_{v i} \neq \Delta_{v^{\prime}} \neq f\left(\Delta_{v^{\prime}}\right) \neq \Delta_{v i}$. But $f$ transforms $\Delta_{v i}$ identically, hence $f(\Delta)=\Delta$ and consequently the simplex $f\left(\Delta_{v^{\prime}}\right)$ is also incident to $\Delta$. But this is impossible because only $\Delta_{v i}$ and $\Delta_{v i^{\prime}}$ among positively oriented simplexes of $T_{\text {, }}$ are incident to $\Delta$.
Hence $\lambda_{r}$ is a cycle and also $\varkappa_{p}+f\left(\varkappa_{y}\right)$ is a cycle. It follows that (30)

$$
f\left(\partial x_{p}\right)=-\partial x_{y} .
$$

Now let $\varepsilon$ be an arbitrary positive number. By the lemma of section 9 there exists an $\eta>0$ and a homomorphism $\vartheta_{0}$ assigning to every $\eta$-chain of dimension $l \leqslant n$ lying in $X$ an $(l+1)$-dimensional $\varepsilon$-chain $\vartheta_{0}(x)$ lying in $\Phi(X)$ and satisfying, for $\varphi$ constituting the identity, conditions (17), (18) and (19). But for almost all $\nu$ the chain $x_{y}$ is an $\eta$-chain. Consider the chain $\vartheta_{0}\left(\varkappa_{r}\right)$. By $(17)$ we have

Let us show that

$$
\partial\left[\vartheta_{0}\left(x_{y}\right)\right]=x_{p}-f\left(x_{v}\right)-\vartheta_{0}\left(\partial x_{p}\right) .
$$

$$
\vartheta_{0}\left(\partial x_{v}\right) \equiv 0 \bmod 2 .
$$

Since $\varkappa_{r}$ is the sum of some $(n-1)$-dimensional simplexes of the triangulation $T_{v}$ of the pseudo-manifold $X$, the chain $\partial \psi_{v}$ is of the form

$$
\text { ' } \partial x_{v}=\Delta_{1}^{\prime}+\Delta_{2}^{\prime}+\ldots+\Delta_{r}^{\prime},
$$

where $\Delta_{1}^{\prime}, \Delta_{2}^{\prime}, \ldots, \Delta_{r}^{\prime}$ are $(n-2)$-dimensional simplexes of $T_{p}$. It follows by (30) that for every $1 \leqslant j \leqslant r$ there exists a $1 \leqslant j^{\prime} \leqslant r$ such that

$$
f\left(\Delta_{j}^{\prime}\right)=-\Delta_{j^{\prime}}^{\prime}
$$

Since the triangulation $T$, is consistent with the involution $f$, we have $f(\Delta) \neq-\Delta$ for every simplex $\Delta$ of $T_{v}$. It follows that the indices $j, j^{\prime}$, satisfying (33), are always different. We conclude that $\partial x_{\text {, can }}$ be represented in the form

Applying (18) we infer that

$$
\partial \varkappa_{p}=\mu_{p}-f\left(\mu_{v}\right) \text {. }
$$

$$
\vartheta_{0}\left(\partial x_{\nu}\right)=\vartheta_{0}\left(\mu_{\nu}\right)-\vartheta_{0} f\left(\mu_{v}\right)=2 \vartheta_{0}\left(\mu_{v}\right)
$$

and consequently (32) holds.

It follows by (31) that

$$
x_{p}-f\left(x_{p}\right) \equiv \partial\left[\vartheta_{0}\left(x_{p}\right)\right] \bmod 2,
$$

and consequently $\gamma_{p}^{\prime}=x_{p}-f\left(x_{p}\right)$ is a cycle modulo 2 homologous to 0 in $\Phi(X)$. By $(29)$ the $(n-1)$-dimensional cycle $x_{p}-f\left(x_{y}\right) \neq 0 \bmod 2$ and consequently $x_{p}-f\left(x_{p}\right)+0 \bmod 2$ in $T_{p}$.

It follows that the $(n-1)$-dimensional cycles $\gamma_{v}^{\prime}$ (modulo 2) constitute a true $(n-1)$-dimensional cycle $\gamma^{\prime}=\left\{\gamma_{v}^{\prime}\right\}$ modulo 2 , not homologous to zero in $X$, but homologous to zero in $\Phi(X) \subset E_{n}$. It follows that the bounded component of $E_{n}-X$ is contained in $\Phi(X)$. Thus the theorem is proved. 
In particular, it follows that if we join every point $x$ of the $(n-1)$ -dimensional sphere $S$, lying in $E_{n}$, with its antipode $x^{*}$ by an acyclic continuum $\Phi(x)=\Phi\left(x^{*}\right)$ and if $\Phi(x)$ constitute a family over $S$, then the interior region of $S$ is swept out by the sets $\Phi(x)$.

\section{References}

[1] K. Borsuk, Sur un espace des transformations continues et ses applications topologiques, Monatsh. für Math. u. Phys. 38 (1931), p. 381-386.

[2] B. Eckmann, Zur Homotopietheorie gefaserter Räume, Comm. Math. Helvetici 14 (1941), p. 141-192.

[3] S. Eilenberg and D. Montgomery, Fixed point theorems for multi-valued transformations, American Journal of Mathematics 68 (1946), p. 214-222.

[4] S. Gołąb, Cn théorème sur le balayage, Fund. Math. 12 (1928), p. 4-19.

[5] F. Hausdorff, Grundzüge der Mengenlehre, Leipzig 1914.

[6] W. Hurewicz, Beiträge zur Topologie der Deformationen III. Klassen und Homologietypen von Abbildungen, Proceedings Ákad. Amsterdam 39 (1936), p. 117-125.

[7] C. Kuratowski, Topologie II, Warszawa 1952.

[8] F. Leja, Un lemme topologique et son application dans la théorie des groupes abstraits, Fund. Math. 10 (1927), p. 421-426:

[9] L. Pon trjagin, The general topological theorem of duality for closed sets, Annals of Math. 35 (1934), p. 904-914.

[10] N. Steenrod, The Topology of Fibre Bundles, Princeton 1951.

Resu par la Rédaction le 16.8.1954

\section{Examples of sets \\ definable by means of two and three quantifiers}

by

\section{A. Mostowski (Warszawa)}

There are many categories of mathematical papers. On the one hand we have first-class papers which are read with interest by many mathematicians and which further the development of mathematical thought. On the other hand we have also papers which are studied exclusively by referees appointed for that task by editors of bibliographical journals and which even by these casual readers are put aside with a sigh "why do these people publish so much?"

The present paper belongs to the second rather than to the first category. I have collected in it a number of very special results which belong to the theory of recursive functions. More explicitly I consider fractions of the form $10^{-x} \alpha(x, y)$ where $a$ is a primitive recursive function and investigate the set of those integers $y$ for which $\lim 10^{-x} \alpha(x, y)$ exists and belongs to a preassigned class of real numbers. $A \stackrel{x}{\text { typical result }}$ is given in the following theorem (cf. theorem 7 below): The set $Z_{a}^{(5)}$ of those $y$ 's for which $\lim 10^{-x} \alpha(x, y)$ exists and is integral is the most general set of the class $Q_{3}^{(1)}, i$. e., the most general set definable in the form $E \prod_{v} \Sigma \prod_{w} R(y, u, v, w)$ with a recursive $R$. The expression "most general." means that if $\alpha$ runs over the set of primitive recursive functions, then the set $Z_{a}^{(5)}$ runs over the whole class $\boldsymbol{Q}_{\mathbf{3}}^{(\mathbf{1})}$.

Investigating this example and other similar ones I encountered some phenomena which I found interesting. If, for example, we narrow down the variability of $\alpha$ 's to the set of functions for which $\lim 10^{-x} \alpha(x, y)$ always exists (i.e., exists for $y=0,1,2, \ldots)$, then the corresponding sets $Z_{d}^{(5)}$ cease to represent arbitrary sets of the class $Q_{3}^{(1)}$. As $\alpha$ runs over the narrower class of functions, the set $Z_{a}^{(5)}$ runs over the whole class $\boldsymbol{Q}_{2}^{(1)}$ which is known to be different from $Q_{3}^{(1)}$. No such reduction occurs if in stead of $Z_{a}^{(5)}$ we consider sets $Z_{a}^{(6)}$ containing all such $y$ 's for which $\lim _{x} 10^{-x} \alpha(x, y)$ exists and is irrational. In this case the set $Z_{a}^{(6)}$ runs 